\title{
Razumikhin-type Theorem for Stochastic Functional Differential Systems via Vector Lyapunov Function
}

\author{
Lei Liu ${ }^{a, b}$, Xuerong $\mathrm{Mao}^{c}$, Jinde $\mathrm{Cao}^{a, *}$ \\ ${ }^{a}$ School of Automation, Southeast University, Nanjing, 210096, China \\ ${ }^{b}$ College of Science, Hohai University, Nanjing, 210098, China \\ ${ }^{c}$ Department of Statistics and Modelling Science, University of Strathclyde, Glasgow G1 1XH, UK.
}

\begin{abstract}
This paper is concerned with input-to-state stability of SFDSs. By using stochastic analysis techniques, Razumikhin techniques and vector Lyapunov function method, vector Razumikhin-type theorem has been established on input-tostate stability for SFDSs. Novel sufficient criteria on the $p$ th moment exponential input-to-state stability are obtained by the established vector Razumikhin-type theorem. When input is zero, an improved criterion on exponential stability is obtained. Two examples are provided to demonstrate validity of the obtained results.
\end{abstract}

Keywords: Vector Lyapunov function, Razumikhin-type theorem, Input-to-state stability, Exponential stability, Stochastic functional differential systems

\section{Introduction}

The stochastic functional differential systems (SFDS), including stochastic delay differential systems (SDDSs), have been widely used since stochastic modelling plays an important role in many branches of science and engineering. During the last decade, stability analysis of SFDSs have received a lot of attention, see, e.g., [7], [8], [12], [16], [21] and references therein. It is well known that dynamical behaviors of SFDSs are often affected by disturbances such as, control, external inputs. In order to investigate how the system dynamic affected by external inputs, the concept of input-to-state stability (ISS) has been originally proposed by Sontag [23], which has played a significant role in control synthesis of nonlinear systems with external inputs. Consequently, the ISS properties for SFDS with external inputs have been studied by many works, see, e.g., [5], [24], [30].

It is well known that Lyapunov method is powerful in stability analysis. The stability conditions based on the Lyapunov functional is very severe restrictions, and it is not very convenient in applications. In order to overcome such difficulty, Razumikhin has evolved Lyapunov method, and established the Razumikhintype theorem for functional systems, which avoid using

\footnotetext{
* Corresponding Author.

E-mail addresses: jdcao@seu.edu.cn
}

Lyapunov functional in stability analysis for functional systems. Moreover, many stability criteria described by differential operator inequalities can be obtained by Razumikhin-type theorems. Hence, Razumikhin-type theorems have been evolved by many authors for various systems. In the past 20 years, Mao and his collaborators have established the a number of Razumikhintype theorems on moment exponential stability for various SFDSs, the reader is referred to [12]-[14], [26] and references therein. For ISS case, Huang et.al established Razumikhin-type theorems on asymptotic for SFDSs in [2]. For more detailed understanding on this topic, please refer to [6], [18], [19].

However, most of the Razumikhin-type theorem on ISS and exponential stability in the existing literature are based on scalar Lyapunov function (SLF). The stability criteria induced by existing Razumikhin-type theorems are described by scalar differential operator inequalities. But many well-known systems are highdimensional, such as, neural networks systems, populations systems. That is to say, there is a gap between the structure of the high-dimensional systems and scalar differential operator inequalities. In order to conquer the gap, many elementary inequalities and matrix inequalities are applied to narrow the gap, which might give rise to more conservatism.

Recently, more and more attentions have been paid to investigate the stability of neural networks via the vec- 
tor Lyapunov function (VLF) method (see [4], [9], [22], [25], [27]-[29]). Most of the them are based on comparison systems with a M-matrix structure. By establishing a vector Halandy inequality with the structure compatible with recurrent neural networks, Li and Liu [4] have improved the stability criteria on the ISS for neural networks with Markovian switching. Two reasons can attributed to it: 1) The coefficient structure of some realistic systems are similar with M-matrix, such as Hopfield neural networks systems, generalized ecological system; 2)The eigenvalue condition of M-matrix is easy to verify. Motivated by the above mentioned literature, the first aim of this paper is to establish a vector Razumikhin-type theorem based on a vector vector operator with a structure of M-matrix. By using Ito formula, Razumikhin techniques and M-matrix theorem, a vector Razumikhin-type theorem is established. With the help of the vector Razumikhin type theorem, several sufficient criteria with vector $\mathscr{L}$-operator differential inequality on $p$-th moment exponential ISS are derived.

Moreover, when the external input is zero, the ISS becomes the traditional Lyapunov stability based on the equilibrium point. As a result, the criteria on ISS become some sufficient conditions on Lyapunov stability. Thus, the second motivation of this paper is to investigate exponential by the established vector Razumikhin type theorem. A sufficient criteria on exponential stability has proposed in this paper, which has slightly improved the results in existing literature [11], [17], [21].

\section{Preliminaries}

Throughout this paper, unless otherwise specified, let $\left(\Omega, \mathscr{F},\left\{\mathscr{F}_{t}\right\}_{t \geq 0}, \mathbb{P}\right)$ be a complete probability space with a filtration $\left\{\mathscr{F}_{t}\right\}_{t \geq 0}$ satisfying the usual conditions (i.e., it is increasing and right continuous while $\mathscr{F}_{0}$ contains all $\mathbb{P}$-null sets). Let $B(t)=\left(B^{1}(t), \cdots, B^{m}(t)\right)$ be an $m$-dimensional Brownian motion defined on the probability space. Let $R_{+}=[0,+\infty)$. Let $\tau>0$ and $C=C_{\mathscr{F}_{0}}^{b}\left([-\tau, 0] ; R^{n}\right)$ the family of all bounded, $\mathscr{F}_{0}$-measurable, $C\left([-\tau, 0] ; R^{n}\right)$-valued, $\mathscr{F}_{t}$-adapted stochastic processes. Let $C\left(R^{n} ; R_{+}\right)$denote the family of all nonnegative functions $w(x)$ on $R^{n}$. For $t>$ 0 , denote by $L_{\mathscr{F}_{t}}^{w}\left([-\tau, 0] ; R^{n}\right)$ the family of all $\mathscr{F}_{t^{-}}$ measurable $C\left([-\tau, 0] ; R^{n}\right)$-valued random variables $\varphi$ such that $\sup _{-\tau \leq \theta \leq 0} E|w(\varphi(\theta))|<\infty$, and let $L_{\mathscr{F}_{t}}^{p}=$ $L_{\mathscr{F}_{t}}^{|x|^{p}}\left([-\tau, 0] ; R^{n}\right)$ for simplicity. Let $\mathscr{L}_{\infty}^{n}$ denote the class of essential bounded functions $u$ from $[0, \infty)$ to $R^{n}$ with $\|u\|_{\infty}=\sup _{t \geq 0}|x(t)|<\infty$. A function $\chi: R_{+} \rightarrow R_{+}$ is said to be class of $\mathscr{K}$ if it is continuous and strictly increasing and satisfies $\chi(0)=0$; it is class of $\mathscr{K}_{\infty}$ if in addition $\chi(s) \rightarrow \infty$ as $s \rightarrow \infty$. Let $G$ be a vector or matrix. By $G \geq 0$ we mean that each element of $G$ is non-negative. By $G \gg 0$ we mean that all elements of $G$ are positive.

Moreover, we also adopt here the traditional notation by letting $Z^{\mathrm{n} \times \mathrm{n}}=\left\{A=\left(a_{i j}\right)_{\mathrm{n} \times \mathrm{n}}: a_{i j} \leq 0, i \neq j\right\}$. A matrix $A \in Z^{\mathrm{n} \times \mathrm{n}}$ is said to be a nonsingular M-matrix, if there exists $x \gg 0$ in $R^{n}$ such that $A x \gg 0$. For a singular Mmatrix $A$, we denote $\Omega_{M}(A)=\left\{x \in R^{n} \mid A x \gg 0, x \gg 0\right\}$.

We consider the following SFDS with external inputs:

$$
\mathrm{d} x=F\left(t, x_{t}, u\right) d t+G\left(t, x_{t}, u\right) d B(t)
$$

with $x_{t}=\{x(t+\theta):-\tau \leq \theta \leq 0\}$ and the given initial data $\xi \in C_{\mathscr{F}_{0}}^{b}\left([-\tau, 0] ; R^{n}\right)$, where

$$
F: R_{+} \times C \times R^{n} \rightarrow R^{n}, G: R_{+} \times C \times R^{n} \rightarrow R^{m n} .
$$

Let $C^{1,2}\left(R_{+} \times R^{n} ; R_{+}\right)$denote the family of all nonnegative functions $W(x, t)$ on $R^{n} \times R_{+}$which are continuously twice differentiable in $u$ and once in $t$. For each $W \in C^{1,2}\left(R_{+} \times R^{n} ; R_{+}\right)$, denote an operator $\mathscr{L} W$ from $R_{+} \times C \times R^{n}$ to $R$ by

$$
\begin{array}{r}
\mathscr{L} W(t, \varphi, u)=W_{t}(t, \varphi(0))+W_{x}(t, \varphi(0)) F(t, \varphi, u) \\
+\frac{1}{2} \operatorname{trace}\left[G^{T}(t, \varphi, u) W_{x x}(t, \varphi(0)) G(t, \varphi, u)\right] .
\end{array}
$$

Definition 2.1: For $p>0$, the system (1) is said to be $p$ th moment exponentially input-to-state stable, if for every $\xi \in L_{\mathscr{F}_{t}}^{p}$ and $u \in \mathscr{L}_{\infty}^{n}$, there exist scalar $L>0, \gamma_{p}>0$ and $\chi \in \mathscr{K}_{\infty}$, such that

$$
E|x(t)|^{p} \leq L \sup _{-\tau \leq \theta \leq 0} E\|\xi(\theta)\|^{p} \exp \left\{-\gamma_{p} t\right\}+\chi\left(\|u\|_{\infty}^{p}\right) .
$$

\section{Main results}

In this section, we will establish the vector Razumikhin-type theorem for SFDS (1), and we assume that for any initial data $\xi \in C_{\mathscr{F}_{0}}^{b}\left([-\tau, 0] ; R^{n}\right)$, system(1) have a unique continuous solution on $t \geq 0$ denoted by $x(t ; \xi)$.

\subsection{The Razumikhin-type Theorem for SFDS}

For stochastic functional differential system (1) without external input

$$
\mathrm{d} x=F\left(t, x_{t}\right) d t+G\left(t, x_{t}\right) d B(t)
$$

Kolmanovskii and Nosov establish the following stability criteria in [37]. However, it is not very convenient in application since the construction of a Lyapunov 
functional is much more harder than that of a Lyapunov function. Thus scholars would like to study the stability of SFDSs using Lyapunonv function. In order for $\mathscr{L} W(t, \varphi, 0)$ to be negative for all function $\varphi \in C$ and $t \geq 0$, some rigorous conditions would be imposed on functions $F(t, \varphi)$ and $G(t, \varphi)$. As a result, the point $\varphi(0)$ must play a dominant role and, therefore, the stability criteria might apply only to equations that are very similar to stochastic differential equations. Fortunately, it is Razumikhin who first indicate that it is unnecessary to verify all $\varphi \in C$ when studying the deterministic differential delay equation. In the past 20 years, Mao and his collaborator generalize the original ideal with Razumikhin to SFDSs. Now we presented the vector Razumikhin-type theorem for SFDSs with external input as following.

Theorem 3.1: Let $\Lambda=\operatorname{diag}\left(\mu_{1}, \cdots, \mu_{n}\right) \gg 0, A=$ $\left(a_{i j}\right)_{n \times n} \geq 0$ be real matrix. Assume that $\Lambda-A$ is a nonsingular M-matrix, and there exist $r \geq 1, Q=$ $\operatorname{diag}\left(q_{1}, \cdots, q_{n}\right) \gg 1, W_{1}, \cdots, W_{n} \in C^{1,2}\left(R_{+} \times R^{n} ; R_{+}\right)$, $w \in C\left(R^{n} ; R_{+}\right), v \in \mathscr{K}$ such that for all

$$
w(x) \leq \sum_{i=1}^{n} W_{i}(t, x) \leq v(x), t \geq 0
$$

and, moreover for all $i=1, \cdots, n$

$$
\begin{array}{rl}
E & \mathscr{L} W_{i}(t, \varphi, u) \leq-\mu_{i} E W_{i}(t, \varphi(0)) \\
& +\sum_{j=1}^{n} a_{i j}\left(E W_{i}(t, \varphi(0))\right)^{1 / r^{\prime}}\left(E W_{j}(t, \varphi(0))\right)^{1 / r} \\
& +\left(E W_{i}(t, \varphi(0))\right)^{1 / r^{\prime}}\left|u_{i}(t)\right|,
\end{array}
$$

for all $t \geq 0$, and those $\varphi \in L_{\mathscr{F}_{t}}^{w}\left([-\tau, 0] ; R^{n}\right)$ satisfying

$$
E W_{i}(t+\theta, \varphi(\theta)) \leq q_{i} E W_{i}(t, \varphi(0)),
$$

on $\theta \in[-\tau, 0]$, where $r^{\prime}=\left(1-r^{-1}\right)^{-1}$. Then the global solution $x(t, \xi)$ to system (1) has the following property:

$$
\begin{aligned}
& \left(E W_{i}(t, x(t))\right)^{1 / r} \\
& \quad \leq \alpha_{i} \Xi^{1 / r} \exp \{-\gamma t\}+\beta_{i}^{t}, i=1, \cdots, n,
\end{aligned}
$$

where $\gamma=\min _{1 \leq i \leq n} \gamma_{i}, r \gamma_{i}=\frac{\log q_{i}}{\tau} \wedge \alpha_{i}^{-1}\left(\mu_{i} \alpha_{i}-\right.$ $\left.\sum_{j=1}^{n} a_{i j} \alpha_{j}\right), \Xi=\sup _{-\tau \leq \theta \leq 0} \sum_{i=1}^{n} E W_{i}(\theta, x(\theta))$, and $\alpha \in$ $\Omega_{M}(\Lambda-A)$ with $\min _{1 \leq i \leq n}\left\{\alpha_{i}\right\} \geq 1, \beta^{t}=\left(\beta_{1}^{t}, \cdots, \beta_{n}^{t}\right)^{T}=$ $(\Lambda-A)^{-1} u^{t}$ with $u^{t}=\left(u_{1}^{t}, \cdots, u_{n}^{t}\right)^{T}, u_{i}^{t}=\sup _{0 \leq s \leq t}\left|u_{i}(s)\right|$. Proof: Since $\Lambda-A$ is a nonsingular M-matrix, there exists $\alpha \in \Omega_{M}(\Lambda-A)$ with $\min _{1 \leq i \leq n}\left\{\alpha_{i}\right\} \geq 1$. In order to prove (6), it is sufficient to show for any $\lambda \in(0, \gamma)$

$$
\begin{aligned}
\left(E W_{i}(t, x(t))\right)^{1 / r} \leq & \alpha_{i} \Xi^{1 / r} \exp \{-\lambda t\} \\
& +\beta_{i}^{t}, \quad i=1, \cdots, n
\end{aligned}
$$

Let $W_{i}(t)=W_{i}(t, x(t)), U_{i}(t)=E W_{i}(t, x(t)), H_{i}(t)=$ $\alpha_{i} \Xi^{1 / r} \exp \{-\gamma t\}$ for simplicity. Since $\xi \in C$, we can find a integer $k_{0}$ such that $\|\xi\|<k_{0}$, a.s. Thus the right side of inequality gives that for any $t \in[-\tau, 0]$

$$
E W_{i}(t, x(t)) \leq E v(|x(t)|) \leq v\left(k_{0}\right), \quad i=1, \cdots, n .
$$

Let $\varrho_{k}^{i}=\inf \left\{t>0 ; E W_{i}(t, x(t))>k\right\}, \varrho_{e}^{i}=\lim _{t \rightarrow \infty} \varrho_{k}^{i}$ and $\varrho_{e}=\wedge_{i=1}^{n} \varrho_{e}^{i}$. It follows from Lemma 3.1 in [2] that $E W_{i}(t, x(t))$ are continuous on $\left[-\tau, \varrho_{e}^{i}\right)$. It is obvious that

$$
W_{i}(0)=E W_{i}(0, x(0)) \leq \alpha_{i}^{r} \Xi \leq\left(\alpha_{i} \Xi^{1 / r}+\beta_{i}^{0}\right)^{r} .
$$

We claim that (7) holds for all $t \in\left[-\tau, \varrho_{e}\right)$. Otherwise, by the continuity of $E W_{i}(t, x(t))$ there exist $\bar{i}$ and the smallest $t^{*}$ such that

$$
\begin{aligned}
& U_{i}(t) \leq\left(H_{i}(t)+\beta_{i}^{t}\right)^{r}, t \in\left[-\tau, t^{*}\right], i=1, \cdots, n, \\
& U_{\bar{i}}\left(t^{*}\right)=\left(H_{\bar{i}}\left(t^{*}\right)+\beta_{\bar{i}}^{t^{*}}\right)^{r}, \\
& D^{+} U_{\bar{i}}\left(t^{*}\right) \geq D^{+}\left(H_{\bar{i}}\left(t^{*}\right)+\beta_{\bar{i}}^{t^{*}}\right)^{r} .
\end{aligned}
$$

By condition (8), for any $\theta \in[-\tau, 0]$

$$
\begin{aligned}
& E W_{i}\left(t^{*}+\theta\right) \leq\left(H_{i}\left(t^{*}+\theta\right)+\beta_{i}^{t^{*}+\theta}\right)^{r} \\
& \quad \leq\left(\alpha_{i} \Xi^{1 / r} \exp \left\{-\lambda\left(t^{*}-\tau\right)\right\}+\beta_{i}^{t^{*}}\right)^{r}<\infty .
\end{aligned}
$$

This implies

$$
\begin{aligned}
& \sup _{-\tau \leq \theta \leq 0} E w\left(x\left(t^{*}+\theta\right)\right) \\
& \quad \leq \sum_{i=1}^{n}\left(\alpha_{i} \Xi^{1 / r} \exp \left\{-\lambda\left(t^{*}-\tau\right)\right\}+\beta_{i}^{t^{*}}\right)^{r}<\infty .
\end{aligned}
$$

which means $x_{t^{*}} \in L_{\mathscr{F}_{t}}^{w}\left([-\tau, 0] ; R^{n}\right)$. From the definition of $\gamma$ and (9), we have

$$
\begin{aligned}
& \left(E W_{\bar{i}}\left(t^{*}+\theta\right)\right)^{1 / r}-\beta_{\bar{i}}^{t^{*}+\theta} \\
& \quad \leq \alpha_{\bar{i}} \Xi^{1 / r} \exp \left\{-\lambda\left(t^{*}+\theta\right)\right\} \\
& \quad \leq q_{\bar{i}}^{1 / r}\left(\left(E W_{\bar{i}}\left(t^{*}\right)^{1 / r}-\beta_{\bar{i}}^{t^{*}}\right) .\right.
\end{aligned}
$$

This implies

$$
\begin{gathered}
E W_{\bar{i}}\left(t^{*}+\theta\right) \leq\left(q_{\bar{i}}^{1 / r}\left(E W_{\bar{i}}\left(t^{*}\right)\right)^{1 / r}\right. \\
\left.+\left(\beta_{\bar{i}}^{t^{*}+\theta}-q_{\bar{i}} \beta_{\bar{i}}^{t^{*}}\right)\right)^{r} \leq q_{\bar{i}} E W_{\bar{i}}\left(t^{*}\right) .
\end{gathered}
$$


We hence see from condition (5) and (8), (9) that

$$
\begin{aligned}
E \mathscr{L} & W_{\bar{i}}\left(t^{*}, x_{t^{*}}, u\right) \\
\leq & -\mu_{\bar{i}} E W_{\bar{i}}\left(t^{*}\right)+\left(E W_{\bar{i}}\left(t^{*}\right)^{1 / r^{\prime}}\left|u_{\bar{i}}\left(t^{*}\right)\right|\right. \\
& +\sum_{j=1}^{n} a_{\bar{i} j}\left(E W_{\bar{i}}\left(t^{*}\right)\right)^{1 / r^{\prime}}\left(E W_{j}\left(t^{*}\right)\right)^{1 / r} \\
\leq & -\mu_{\bar{i}}\left(H_{\bar{i}}\left(t^{*}\right)+\beta_{\bar{i}}^{t^{*}}\right)^{r} \\
& +\left(H_{\bar{i}}\left(t^{*}\right)+\beta_{\bar{i}}^{t^{*}}\right)^{r-1}\left|u_{\bar{i}}\left(t^{*}\right)\right| \\
& +\sum_{j=1}^{n} a_{\bar{i} j}\left(H_{\bar{i}}\left(t^{*}\right)+\beta_{\bar{i}}^{t^{*}}\right)^{r-1}\left(H_{j}\left(t^{*}\right)+\beta_{j}^{t^{*}}\right) \\
\leq & \left(H_{\bar{i}}\left(t^{*}\right)+\beta_{\bar{i}}^{t^{*}}\right)^{r-1}\left(-\mu_{\bar{i}} H_{\bar{i}}\left(t^{*}\right)+\sum_{j=1}^{n} a_{\bar{i} j}\right. \\
& \left.\times H_{j}\left(t^{*}\right)+\sum_{j=1}^{n} a_{\bar{i} j} \beta_{j}^{t^{*}}-\mu_{\bar{i}} \beta_{\bar{i}}^{t^{*}}+\left|u_{\bar{i}}\left(t^{*}\right)\right|\right) .
\end{aligned}
$$

The definition of $\gamma$ and $\beta^{t}$ imply easily that

$$
\begin{aligned}
& -\mu_{\bar{i}} H_{\bar{i}}\left(t^{*}\right)+\sum_{j=1}^{n} a_{\bar{i} j} H_{j}\left(t^{*}\right) \\
& \leq \Xi^{1 / r} \exp \left\{-\lambda t^{*}\right\}\left(-\mu_{\bar{i}} \alpha_{\bar{i}}+\sum_{j=1}^{n} a_{\bar{i} j} \alpha_{j}\right) \\
& \quad<-r \lambda H_{\bar{i}}\left(t^{*}\right), \\
& -\mu_{\bar{i}} \beta_{\bar{i}}^{t^{*}}+\sum_{j=1}^{n} a_{\bar{i} j} \beta_{j}^{t^{*}}+x_{\bar{i}}\left(t^{*}\right) \leq 0 .
\end{aligned}
$$

By virtue of the Definition of Dini-derivative, we have $D^{+} E W_{\bar{i}}\left(t^{*}, x\left(t^{*}\right)\right)=E \mathscr{L} W_{\bar{i}}\left(t^{*}, x_{t^{*}}, u\right)$. Submitting (12) and (13) into (11) yields

$$
\begin{array}{rl}
E & \mathscr{L} W_{\bar{i}}\left(t^{*}, x_{t^{*}}, u\right)=D^{+} E W_{\bar{i}}\left(t^{*}, x\left(t^{*}\right)\right) \\
& <-\lambda r H_{\bar{i}}\left(t^{*}\right)\left(H_{\bar{i}}\left(t^{*}+\beta_{\bar{i}}^{t^{*}}\right)\right)^{r-1} \\
& =r\left(H_{\bar{i}}\left(t^{*}\right)+\beta_{\bar{i}}^{t^{*}}\right)^{r-1} D^{+} H_{\bar{i}}\left(t^{*}\right) \leq D^{+}\left(H_{\bar{i}}\left(t^{*}\right)+\beta_{\bar{i}}^{t^{*}}\right)^{r},
\end{array}
$$

which contradicts with (10). Therefor for any $t \in$ $\left[-\tau, \varrho_{e}\right),(7)$ holds. Now we process to show $\varrho_{e}=+\infty$. Otherwise, there exits $i^{* *}$ such that $\varrho_{e}^{i^{* *}}<+\infty$. Letting $t \rightarrow \varrho_{e}^{i^{* *}}$ on both side of (6) yields

$$
\begin{aligned}
\infty & =\lim _{t \rightarrow \varrho_{e}^{i^{* *}}}\left(E W_{i^{* *}}(t, x(t))\right)^{1 / r} \\
& \leq \alpha_{i^{* *}} \Xi^{1 / r} \exp \left\{-\gamma \varrho_{e}^{i^{*^{* *}}}\right\}+\beta_{i^{* *}}^{e_{e}^{i^{* *}}}<\infty .
\end{aligned}
$$

which is a contradiction. Thus the inequality (6) holds for any $t \geq 0$. The proof is completed.
Remark 1: When $r=1, w(x)=|x|^{p}, u(t)=0$ and using the SLF, the Theorem 3.1 becomes the classical Razumikhin-type theorems that established for SFDSs by Mao and his collaborators(see [12]-[14]). Hence, the classical Razumikhin-type theorems on exponential stability are generalized to vector form for SFDSs.

Remark 2: For the stochastic differential system, the boundedness and continuity of $E W_{i}(t, x(t))$ is of vital importance, which are guaranteed by linear growth conditions (LGC) and power function type bilateral condition posed on Lyapunov function, see [12]-[? ]. In this paper, the LGC is no longer needed and the bilateral condition has been replaced by a weaker (4). In order to establish the Razumikhin-type Theorem 3.1 in the absent of there two conditions, we proved the Theorem 3.1 by three steps. Firstly, by virtue of Lemma 3.1 in [2] and the boundedness of $\xi \in C$, we showed that $E W_{i}(t, x(t))$ are continuous on $\left[-\tau, \varrho_{e}^{i}\right)$. Secondly, with the aid of classical Razumikhin technique, we proved that inequality (6) holds on $\left[-\tau, \varrho_{e}\right)$. Finally, we proved by contradiction that inequality (6) holds for all $t \geq-\tau$, which also ensured the continuous of $E W_{i}(t, x(t))$.

\subsection{Exponential input-to-state stability}

Now we process to apply the vector Razumikhin-type theorem to deal with the exponential ISS of the SFDS (1).

Theorem 3.2: Let the condition (4) of Theorem 3.1 hold. Assume that $\Lambda=\operatorname{diag}\left(\mu_{1}, \cdots, \mu_{n}\right) \gg 0, A=\left(a_{i j}\right)_{n \times n} \geq$ $0, B=\left(b_{i j}\right)_{n \times n} \geq 0$, are real matrix. Assume that there exist $r \geq 1$ and $W_{1}, \cdots, W_{n} \in C^{1,2}\left(R_{+} \times R^{n} ; R_{+}\right)$as well as probability measures $\eta_{1}, \cdots, \eta_{n}$ on $[-\tau, 0]$ such that for all $i=1, \cdots, n$

$$
\lim _{|x| \rightarrow \infty}\left[\inf _{0 \leq t \leq \infty} \sum_{i=1}^{n} W_{i}(t, x)\right]=\infty,
$$

and

$$
\begin{aligned}
& \mathscr{L} W_{i}(t, \varphi, v) \leq-\mu_{i} W_{i}(t, \varphi(0)) \\
& \quad+\sum_{j=1}^{n} a_{i j}\left(W_{i}(t, \varphi(0))\right)^{1 / r^{\prime}}\left(W_{j}(t, \varphi(0))\right)^{1 / r} \\
& \quad+\left(W_{i}(t, \varphi(0))\right)^{1 / r^{\prime}}\left|u_{i}(t)\right|+\sum_{j=1}^{n} b_{i j}\left(W_{i}(t, \varphi(0))\right)^{1 / r^{\prime}} \\
& \quad \times\left(\int_{-\tau}^{0} W_{j}(t+\theta, \varphi(\theta)) d \eta_{j}(\theta)\right)^{1 / r},
\end{aligned}
$$


for all $t \geq 0$ with $r^{\prime}=\left(1-r^{-1}\right)^{-1}$. We then have the following assertions:

i) Given any initial data $\xi \in C_{\mathscr{F}_{0}}^{b}\left([-\tau, 0] ; R^{n}\right)$, the global solution to the system (1) denoted by $x(t ; \xi)$ satisfys the following property:

$$
\sup _{0 \leq s \leq t} \sum_{i=1}^{n} E W_{i}(s, x(s))<\infty, \quad \forall t \geq 0 .
$$

ii) If $\Lambda-(A+B)$ is a nonsingular M-matrix, the global solution $x(t ; \xi)$ to system (1) has the following property:

$$
\begin{gathered}
\left(E W_{i}(t, x(t))\right)^{1 / r} \leq \alpha_{i} \Xi^{1 / r} \exp \left\{-\gamma_{r} t\right\}+\beta_{i}^{t}, \\
i=1, \cdots, n .
\end{gathered}
$$

where $\beta^{t}=\left(\beta_{1}^{t}, \cdots, \beta_{n}^{t}\right)^{T}$ and $\Xi$ are defined in Theorem 3.1, and $\gamma_{r}=\sup \left\{\theta>0, \alpha_{i} \theta-r^{-1} \mu_{i} \alpha_{i}+\right.$ $\left.r^{-1} \sum_{j=1}^{n} a_{i j} \alpha_{j}+e^{\theta \tau} r^{-1} \sum_{j=1}^{n} b_{i j} \alpha_{j}<0, i=1, \cdots, n\right\}$ with $\alpha \in \Omega_{M}(\Lambda-(A+B)), \min _{1 \leq i \leq n}\left\{\alpha_{i}\right\} \geq 1$.

Proof: The proof is composed of two parts. In part 1, we show the continuous of $E W_{i}(t, x(t))$. The assertion ii) is proved by Theorem 3.1 in part 2. Let $x(t)=x(t, \xi), W_{i}(t)=W_{i}(t, x(t)), i=1, \cdots, n$ for simplicity.

Part 1: For sufficiently large number $k$, set $\rho_{k}=\inf \{t \geq$ $\left.0: W_{i}(t, x(t))>k, i=1, \cdots, n\right\}$. Applying the Itô formula to system (1) and using the Hölder inequality yields

$$
\begin{aligned}
E W_{i}( & \left.\wedge \rho_{k}\right) \leq E W_{i}(0) \\
& +\int_{0}^{t} \sum_{j=1}^{n} a_{i j}\left(E W_{j}\left(s \wedge \rho_{k}\right)\right)^{1 / r}\left(E W_{i}\left(s \wedge \rho_{k}\right)\right)^{1 / r^{\prime}} \\
& +\int_{0}^{t} \sum_{j=1}^{n} b_{i j}\left(E W_{i}\left(s \wedge \rho_{k}\right)\right)^{1 / r^{\prime}} \\
& \times\left(\int_{-\tau}^{0} E W_{j}\left((s+\theta) \wedge \rho_{k}\right) d \eta_{j}(\theta)\right)^{1 / r} d s \\
& +\int_{0}^{t}\left(E W_{i}\left(s \wedge \rho_{k}\right)\right)^{1 / r^{\prime}}\left|u_{i}\left(s \wedge \rho_{k}\right)\right| d s .
\end{aligned}
$$

By virtue of the Young inequality

$$
v^{\varsigma} v^{1-\varsigma} \leq \varsigma u+(1-\varsigma) v, \quad \forall u, v \geq 0, \varsigma \in(0,1),
$$

and compute that

$$
\begin{aligned}
& E W_{i}\left(t \wedge \rho_{k}\right) \leq E W_{i}(0) \\
& +\int_{0}^{t} \sum_{j=1}^{n} a_{i j}\left(r^{\prime-1} E W_{i}\left(s \wedge \rho_{k}\right)+r^{-1} E W_{j}\left(s \wedge \rho_{k}\right)\right) d s \\
& \quad+\int_{0}^{t} \sum_{j=1}^{n} b_{i j}\left(r^{\prime-1} E W_{i}\left(s \wedge \rho_{k}\right)\right. \\
& \left.+r^{-1} \sup _{-\tau \leq \theta \leq 0} E W_{j}\left((s+\theta) \wedge \rho_{k}\right)\right) d s \\
& \quad+\int_{0}^{t}\left[\left(r^{\prime-1} E W_{i}\left(s \wedge \rho_{k}\right)\right)^{1 / r^{\prime}}+r^{-1} u_{i}^{r}\left(s \wedge \rho_{k}\right)\right] d s
\end{aligned}
$$

and then

$$
\begin{aligned}
& \sup _{0 \leq t \leq t_{1}} E W_{i}\left(t \wedge \rho_{k}\right) \\
& \leq E W_{i}(0)+r^{-1} \sum_{j=1}^{n} b_{i j} \sup _{-\tau \leq s \leq 0} E W_{j}\left(s \wedge \rho_{k}\right) t_{1} \\
& \quad+E \sum_{j=1}^{n}\left(a_{i j}+b_{i j}\right) \int_{0}^{t_{1}}\left(r^{\prime-1} \sup _{0 \leq s \leq t} W_{i}\left(s \wedge \rho_{k}\right)\right. \\
& \left.+r^{-1} \sup _{0 \leq s \leq t} W_{j}\left(s \wedge \rho_{k}\right)\right) d t \\
& +\int_{0}^{t_{1}}\left(\left(r^{\prime-1} \sup _{0 \leq s \leq t} E W_{i}\left(s \wedge \rho_{k}\right)\right)^{1 / r^{\prime}}\right. \\
& \left.+r^{-1} \sup _{0 \leq s \leq t}\left|u_{i}\left(s \wedge \rho_{k}\right)\right|^{r}\right) d t .
\end{aligned}
$$

Summing $\sup _{0 \leq t \leq t_{1}} E W_{i}\left(t \wedge \rho_{k}\right)$ from 1 to $n$ yields

$$
\begin{aligned}
& \sum_{i=1}^{n} \sup _{0 \leq t \leq t_{1}} E W_{i}\left(t \wedge \rho_{k}\right) \leq \sum_{i=1}^{n} E W_{i}(0) \\
& \quad+\left(r^{-1} \sum_{i=1}^{n} \sum_{j=1}^{n} b_{i j} \sup _{-\tau \leq s \leq 0} E W_{j}\left(s \wedge \rho_{k}\right)\right) t_{1} \\
& \quad+r^{\prime-1} \sum_{i=1}^{n} \sum_{j=1}^{n}\left(a_{i j}+b_{i j}\right) \int_{0}^{t_{1}} \sup _{0 \leq s \leq t} E W_{i}\left(s \wedge \rho_{k}\right) d t \\
& +r^{-1} \sum_{i=1}^{n} \sum_{j=1}^{n}\left(a_{i j}+b_{i j}\right) \int_{0}^{t_{1}} \sup _{0 \leq s \leq t} E W_{j}\left(s \wedge \rho_{k}\right) \\
& \quad+\int_{0}^{t_{1}}\left(\left(r^{\prime-1} \sup _{0 \leq s \leq t} \sum_{i=1}^{n} E W_{i}\left(s \wedge \rho_{k}\right)\right)^{1 / r^{\prime}}\right. \\
& \left.\quad+r^{-1} \sup _{0 \leq s \leq t} \sum_{i=1}^{n}\left|u_{i}\left(s \wedge \rho_{k}\right)\right|^{r}\right) d t .
\end{aligned}
$$


which means

$$
\begin{aligned}
& \sum_{i=1}^{n} \sup _{0 \leq t \leq t_{1}} E W_{i}\left(t \wedge \rho_{k}\right) \leq K+M t_{1} \\
& \quad+\beta \int_{0}^{t_{1}} \sup _{0 \leq s \leq t} \sum_{i=1}^{n} E W_{i}\left(s \wedge \rho_{k}\right) d t .
\end{aligned}
$$

where $\quad \beta=\max _{1 \leq i \leq n}\left(r^{\prime-1} \sum_{j=1}^{n}\left(a_{i j}+\right.\right.$ $\left.\left.b_{i j}+1\right)+r^{-1} \sum_{j=1}^{n}\left(a_{j i}+b_{j i}\right)\right), \quad M=$ $r^{-1}\left(\sum_{i=1}^{n} \sum_{j=1}^{n} b_{i j} \sup _{-\tau \leq s \leq 0} E W_{j}\left(s \wedge \rho_{k}\right)+\sum_{i=1}^{n}\left\|u_{i}\right\|_{p}^{r}\right)$. Setting $l^{(k)}(t)=\sum_{i=1}^{n} \sup _{0 \leq s \leq t} E W_{i}\left(s \wedge \rho_{k}\right),(21)$ can be rewritten as

$$
l^{(k)}\left(t_{1}\right) \leq \Xi+M t_{1}+\int_{0}^{t_{1}} \beta l^{(k)}(t) d t .
$$

The Gronwall inequality implies that

$$
l^{(k)}\left(t_{1}\right) \leq \Xi+M t_{1}+\beta \int_{0}^{t_{1}} \exp \left\{\beta\left(t_{1}-s\right)\right\}(\Xi+M s) d s .
$$

By virtue of the well-known Fatou Lemma, we have

$$
\begin{aligned}
& E\left(\sup _{0 \leq s \leq t} \sum_{i=1}^{n} W_{i}(s, x(s))\right) \\
& =E\left(\sup _{0 \leq s \leq t} \sum_{i=1}^{n} \liminf _{k \rightarrow \infty} W_{i}\left(s \wedge \rho_{k}\right)\right) \\
& \leq E\left(\liminf _{k \rightarrow \infty} \sup _{0 \leq s \leq t} \sum_{i=1}^{n} W_{i}\left(s \wedge \rho_{k}\right)\right) \\
& \leq \liminf _{k \rightarrow \infty} E\left(\sup _{0 \leq s \leq t} \sum_{i=1}^{n} W_{i}\left(s \wedge \rho_{k}\right)\right) \leq \Xi+M t+ \\
& \beta_{1} \int_{0}^{t} \exp \{\beta(t-s)\}(\Xi+M s) d s<\infty, \quad t>0,
\end{aligned}
$$

which implies the required assertion i).

Part 2: Now we begin to prove the assertion ii). The proof consists of two steps. The first step is to indicate the upper bound of $\left(E W_{i}(t, x(t))\right)^{1 / r}-\beta_{i}^{t}, i=1, \cdots, n$ can be controlled by a exponential function. The second step is to show that the exponent should not be greater than $-\gamma_{r}$.

Step 1 of Part 2: Since $\Lambda-(A+B)$ is a nonsingular M-matrix, there exists $\alpha \in \Omega_{M}(\Lambda-(A+B))$ with $\min _{1 \leq i \leq n}\left\{\alpha_{i}\right\} \geq 1$ such that

$$
\mu_{i} \alpha_{i}-\sum_{j=1}^{n} a_{i j} \alpha_{j}-\sum_{j=1}^{n} b_{i j} \alpha_{j}>0, i=1, \cdots, n .
$$

Setting $\beta_{i}=\left(\sum_{j=1}^{n} b_{i j} \alpha_{j}\right)^{-1}\left(\mu_{i} \alpha_{i}-\sum_{j=1}^{n} a_{i j} \alpha_{j}\right)$, for any $q_{i} \in\left(1, \beta_{i}^{r}\right)$, we have

$$
\mu_{i} \alpha_{i}-\sum_{j=1}^{n} a_{i j} \alpha_{j}-q_{i}^{1 / r} \sum_{j=1}^{n} b_{i j} \alpha_{j}>0, i=1, \cdots, n,
$$

which means $\Lambda-(A+Q B)$ is still a nonsingular M-matrix with $Q^{r}=\operatorname{diag}\left\{q_{1}, \cdots, q_{n}\right\}, q_{i} \in\left(1, \beta_{i}^{r}\right), i=1, \cdots, n$. If $t \geq 0, \varphi \in L_{\mathscr{F}_{t}}^{w}\left([-\tau, 0] ; R^{n}\right)$ satisfying

$$
E W_{i}(t+\theta, \varphi(\theta)) \leq q_{i} E W_{i}(t, \varphi(0)), i=1, \cdots, n .
$$

By the condition (15)

$$
\begin{array}{rl}
E & \mathscr{L} W_{i}\left(t, x_{t}, u\right) \leq-\mu_{i} E W_{i}(t, x(t)) \\
& +\left(E W_{i}(t, x(t))\right)^{1 / r^{\prime}}\left|u_{i}(t)\right|+\sum_{j=1}^{n}\left(a_{i j}\right. \\
& \left.+q_{i}^{1 / r} b_{i j}\right)\left(E W_{i}(t, x(t))\right)^{1 / r^{\prime}}\left(E W_{j}(t, x(t))\right)^{1 / r} .
\end{array}
$$

So by Theorem 3.1, the global solution to system (27)

$$
\begin{aligned}
& \left(E W_{i}(t, x(t))\right)^{1 / r} \\
& \quad \leq \alpha_{i} \Xi^{1 / r} \exp \left\{-\gamma^{\prime} t\right\}+\beta_{i}^{t}, i=1, \cdots, n .
\end{aligned}
$$

where $\gamma^{\prime}=\hat{\gamma}_{i}^{\prime}, r \gamma_{i}^{\prime}=\tau^{-1} \log \left(q_{i}\right) \wedge \alpha_{i}^{-1}\left(\mu_{i} \alpha_{i}-\sum_{j=1}^{n}\left(a_{i j}+\right.\right.$ $\left.\left.q_{i} b_{i j}\right)\right)$.

Step 2 of Part 2: Set $f_{i}\left(q_{i}\right)=\tau^{-1} \log q_{i}^{1 / r}$ and $h_{i}\left(q_{i}\right)=$ $\alpha_{i}^{-1}\left(\mu_{i} \alpha_{i}-\sum_{j=1}^{n}\left(a_{i j}+q_{i}^{1 / r} b_{i j}\right)\right), i=1, \cdots, n$. Simple computations show that $f_{i}\left(q_{i}\right)$ is increasing function on $\left(1, \beta_{i}^{r}\right)$ while $h_{i}\left(q_{i}\right)$ are decreasing function for $i=1, \cdots, n$. Combining with the fact that $f_{i}(1)=0<$ $h_{i}(1), f_{i}\left(\beta_{i}^{r}\right)>0=h_{i}\left(\beta_{i}^{r}\right)$, we can claim that there exists a unique $q_{i}^{*}$ such that $\gamma_{i}^{\prime}\left(q_{i}^{*}\right)=f_{i}\left(q_{i}^{*}\right)=h_{i}\left(q_{i}^{*}\right)$. By the definition of $q_{i}^{*}$ we have

$$
\begin{aligned}
\gamma_{i}^{\prime}\left(q_{i}^{*}\right)= & \sup \left\{\tau^{-1} \log q_{i}^{1 / r} \mid \tau^{-1} \log q_{i}^{1 / r}\right. \\
& \left.-r^{-1} \alpha_{i}^{-1}\left(\mu_{i} \alpha_{i}-\sum_{j=1}^{n}\left(a_{i j}+q_{i} b_{i j}\right)\right)<0, q_{i}>0\right\} \\
= & \sup \left\{\theta_{i} \mid \alpha_{i} \theta-r^{-1} \mu_{i} \alpha_{i}+r^{-1} \sum_{j=1}^{n} a_{i j} \alpha_{j}\right. \\
& \left.+e^{\theta \tau} r^{-1} \sum_{j=1}^{n} b_{i j} \alpha_{j}<0, \theta>0\right\} .
\end{aligned}
$$

Moreover, we can claim that

$$
\begin{aligned}
& \gamma_{r}=\sup \left\{\theta>0, \alpha_{i} \theta-r^{-1} \mu_{i} \alpha_{i}+r^{-1} \sum_{j=1}^{n} a_{i j} \alpha_{j}\right. \\
&\left.+e^{\theta \tau} r^{-1} \sum_{j=1}^{n} b_{i j} \alpha_{j}<0, i=1, \cdots, n\right\}=\hat{\gamma}_{i}^{\prime}\left(q_{i}^{*}\right) .
\end{aligned}
$$


It yields the desired assertion ii). The proof is completed.

If $\sum_{i=1}^{n} W_{i}(t, x)$ has a infinity lower bound as $|x|^{p}$, we have the following criteria on $p$ th exponential ISS of SFDS (1).

Corollary 3.1: Let all the assumptions of Theorem 3.2 hold. Moreover, if there exists $C_{1}>0, p>0$ such that

$$
C_{1}|x|^{p} \leq \sum_{i=1}^{n} W_{i}(t, x), t \geq 0 .
$$

Then the system (1) is $p$ th moment exponential ISS.

Proof: It follows from the assertion ii) of Theorem 3.2 that

$$
\begin{gathered}
E W_{i}(t, x(t)) \leq\left(\alpha_{i} \Xi^{1 / r} \exp \{-\gamma t\}+\beta_{i}^{t}\right)^{r} \\
\leq 2^{r-1} \alpha_{i}^{r} \Xi+2^{r-1}\left(\beta_{i}^{t}\right)^{r} .
\end{gathered}
$$

By condition (24), we compute that

$$
\begin{aligned}
E|x(t)|^{p} & \leq C_{1}^{-1} 2^{r-1} \sum_{i=1}^{n} \alpha_{i}^{r} \Xi \exp \{-r \gamma t\} \\
& +C_{1}^{-1} 2^{r-1} \sum_{i=1}^{n}\left[\left(\beta_{i}^{t}\right)^{p}\right]^{r / p} .
\end{aligned}
$$

By virtue of the definition of $\beta^{t}$ that

$$
\begin{aligned}
& \left(\beta_{i}^{t}\right)^{p}=\left(e_{i}(\Lambda-(A+B))^{-1} u^{t}\right)^{p} \\
& \quad \leq\left|e_{i}(\Lambda-(A+B))^{-1}\right|^{p}\left|u^{t}\right|^{p} .
\end{aligned}
$$

Taking the supremum of $\beta_{i}^{p}(t)$ over interval $[0, \infty)$ yields

$$
\sup _{0 \leq t \leq \infty} \beta_{i}^{p}(t) \leq\left|e_{i}(\Lambda-(A+B))^{-1}\right|^{p}\|u\|_{\infty}^{p} .
$$

Submitting (26) into (25) yields

$$
E|x(t)|^{p} \leq L \exp \left\{-r \gamma_{r} t\right\} E\|\xi\|^{p}+\chi\left(|u|_{\infty}^{p}\right),
$$

where $L=C_{1}^{-1} 2^{r-1} \sum_{i=1}^{n} \alpha_{i}^{r}, \chi(t)=C_{1}^{-1} 2^{r-1} \sum_{i=1}^{n} \mid e_{i}(\Lambda-$ $(A+B))^{-1} \mid{ }^{r} t^{r / p}$. The proof is completed.

Remark 3: In fact, the moment boundedness can be guaranteed by those $\mathscr{L} V$ operator whose upper bound may take a much more general form. More detailed understanding on this topic can be referred to [11], [21] and reference therein.

Remark 4: It is worth emphasizing that the vector operator differential inequality (15) in Theorem 3.2 is a generalisation of the existing operator inequalities on exponential stability for SFDSs which are in terms of SLF (see [16]) or VLF (see [27], [29]). Especially, the inequality (15) has several advantage in discussing $p$ th moment exponential stability for stochastically perturbed neural networks and stochastically perturbed generalized ecological systems. When taking $V=|x|^{p}$, the cross-item, such as, $\left|x_{i}\right|^{p-1}\left|x_{j}\right|$ are inevitable encountered. In order to establish the sufficient criteria, the cross-item are amplified by the elementary inequalities. With the help of Theorem 3.2, the amplification might be avoided when discussing these systems. It could be inferred that the vector operator differential inequality (15) has several advantages over those operator differential inequalities in [16], [27], [29] for stochastically perturbed neural networks and stochastically perturbed generalized ecological systems, which can be illustrated by the examples given in Section 4.

Consider an SDDS of the form:

$$
\begin{aligned}
d x(t)= & f(t, x(t), x(t-\tau(t))) d t \\
& +g(t, x(t), x(t-\tau(t))) d B(t),
\end{aligned}
$$

on $t \geq 0$ with initial data $\xi \in C$, where $\tau(\cdot): R_{+} \rightarrow[0, \tau]$ is continuous and

$$
f: R_{+} \times R^{2 n} \times R^{n} \rightarrow R^{n}, g: R_{+} \times R^{2 n} \times R^{n} \rightarrow R^{n \times m} .
$$

If we define $F(t, \varphi)=f(t, \varphi(0), \varphi(-\tau(0))), \quad G(\varphi, t)=$ $g(t, \varphi(0), \varphi(-\tau(0)))$, the system $(27)$ can be seen as a class of system (1).

By virtue of similar technique presented in the Theorem 3.1 and Corollary 3.1, a sufficient criterion on $p$ th moment ISS on SDDSs is obtained as follows:

Corollary 3.2: Let all the assumption of Corollary 3.1 hold expect inequality (15) which is replaced by

$$
\begin{aligned}
& \mathscr{L} W_{i}(t, \varphi, u) \leq-\mu_{i} W_{i}(t, \varphi(0)) \\
& \quad+\sum_{j=1}^{n} a_{i j}\left(W_{j}(t, \varphi(0))\right)^{1 / r^{\prime}}\left(W_{j}(t, \varphi(0))\right)^{1 / r} \\
& \quad+\sum_{j=1}^{n} b_{i j}\left(W_{j}(t, \varphi(0))\right)^{1 / r^{\prime}}\left(W_{j}(t-\tau(t), \varphi(-\tau(0)))\right)^{1 / r} \\
& +\left(W_{i}(t, \varphi(0))\right)^{1 / r^{\prime}}\left|u_{i}(t)\right| .
\end{aligned}
$$

Then the system (27) is $p$ th moment exponential ISS.

\subsection{Exponential stability}

When $u(t) \equiv 0$, the system (1) becomes

$$
d x=F\left(t, x_{t}\right) d t+G\left(t, x_{t}\right) d B(t) .
$$


The $p$ th moment exponential ISS degenerates into the $p$ th moment exponential stability. In this subsection, we assume that $F(t, 0) \equiv 0, G(t, 0) \equiv 0$, and system (29) has a unique trivial solution $x(t ; 0) \equiv 0$. The main aim of this subsection is to study the exponential stability of system (29).

Theorem 3.3: Let all the assumptions of Theorem 3.2 hold and $u(t)=0$. Moreover, if there exists $D>0$ such that for $i=1,2, \cdots, n$

$$
\begin{gathered}
\left|\frac{\partial W_{i}^{\mathrm{T}}}{\partial x}(t, x(t)) G\left(t, x_{t}\right)\right| \leq D \sum_{i=1}^{n}\left(W_{i}(t, x(t))\right. \\
\left.\quad+\int_{-\tau}^{0} W_{i}(t+\theta, x(t+\theta)) d v_{j}(\theta)\right),
\end{gathered}
$$

where $v_{1}(\theta), \cdots, v_{n}(\theta)$ are probability measures on $[-\tau, 0]$. Then we have the following property:

$$
\begin{aligned}
& \limsup _{t \rightarrow \infty} \frac{\log E W_{i}(t, x(t))}{t} \leq-\gamma_{r}, \\
& \limsup _{t \rightarrow \infty} \frac{\log W_{i}(t, x(t))}{t} \leq-\gamma_{r} \text { a.s. } i=1 . \cdots . n .
\end{aligned}
$$

Proof: Let $x(t)=x(t, \xi), G(t)=G\left(t, x_{t}\right), W_{i}(t)=$ $W_{i}(t, x(t)), i=1, \cdots, n$ for simplicity. The property (31) follows by condition (17) with $v(t)=0$ in Theorem 3.2 immediately. Now we proceed to show the property (32). Write $\left\|W_{i, k \tau}\right\|=\sup _{0 \leq \bar{\theta} \leq \tau} W_{i}(k \tau+\bar{\theta})$ for any integer $k>0$. By the Itô's formula, it is easy to show

$$
\begin{aligned}
& W_{i}(k \tau+\bar{\theta})=W_{i}(k \tau) \\
& \quad+\int_{k \tau}^{k \tau+\bar{\theta}} \mathscr{L} W_{i}(s) d s+\int_{k \tau}^{k \tau+\bar{\theta}} \frac{\partial W_{i}^{\mathrm{T}}}{\partial x} G(s) d B(s) \\
& \leq W_{i}(k \tau)+\int_{k \tau}^{k \tau+\bar{\theta}} \sum_{j=1}^{n} a_{i j}\left(W_{j}(s)\right)^{1 / r}\left(W_{i}(s)\right)^{1 / r^{\prime}} d s \\
& \quad+\int_{k \tau}^{k \tau+\bar{\theta}} \sum_{j=1}^{n} b_{i j}\left(\int_{-\tau}^{0} W_{j}(s+\theta) d v_{j}(\theta)\right)^{1 / r} \\
& \quad \times\left(W_{i}(s)\right)^{1 / r^{\prime}} d s+\left|\int_{k \tau}^{k \tau+\bar{\theta}} \frac{\partial W_{i}^{\mathrm{T}}}{\partial x} G(s) d B(s)\right|
\end{aligned}
$$

Making use the Young inequality (stated in Theorem
3.2) with $\varsigma=1 / 2$, we then have

$$
\begin{gathered}
E \sup _{0 \leq \bar{\theta} \leq \tau} W_{i}(k \tau+\bar{\theta}) \leq E W_{i}(k \tau)+\int_{k \tau}^{(k+1) \tau} \sum_{j=1}^{n} \\
\left(r^{\prime-1}\left(a_{i j}+b_{i j}\right) E W_{i}(s)+r^{-1} a_{i j} E W_{j}(s)\right. \\
\left.+r^{-1} b_{i j} \int_{-\tau}^{0} E W_{j}(s+\theta) d \eta_{j}(\theta)\right) d s \\
+E \sup _{0 \leq \bar{\theta} \leq \tau}\left|\int_{k \tau}^{k \tau+\bar{\theta}} \frac{\partial W_{i}^{\mathrm{T}}}{\partial x} G(s) d B(s)\right| .
\end{gathered}
$$

By the Burkholder-Davis-Gundy inequality

$$
\begin{aligned}
& E \sup _{0 \leq \bar{\theta} \leq \tau}\left|\int_{k \tau}^{k \tau+\bar{\theta}} \frac{\partial W_{i}^{\mathrm{T}}}{\partial x} G(s) d B(s)\right| \\
& \leq \sqrt{32} E\left(\int_{k \tau}^{(k+1) \tau}\left|\frac{\partial W_{i}^{\mathrm{T}}}{\partial x} G(s)\right|^{2} d s\right)^{\frac{1}{2}} \\
& \leq \sqrt{32} D E\left(\int _ { k \tau } ^ { ( k + 1 ) \tau } \left(\sum_{j=1}^{n} W_{j}(s)\right.\right. \\
& \left.\left.+\int_{-\tau}^{0} W_{j}(s+\theta) d v_{j}(\theta)\right)^{2} d s\right)^{\frac{1}{2}} \\
& \leq 2 \sqrt{2} D E\left[\varepsilon \operatorname { s u p } _ { 0 \leq \overline { \theta } \leq \tau } \left(\sum_{j=1}^{n} W_{j}(k \tau+\bar{\theta})\right.\right. \\
& \left.+\sup _{-\tau \leq \theta \leq 0}^{n} \sum_{j=1}^{n} W_{j}(k \tau+\bar{\theta}+\theta)\right) \\
& +\varepsilon^{-1} \int_{k \tau}^{(k+1) \tau}\left(\sum_{j=1}^{n} W_{j}(s)\right. \\
& \left.\left.+\sum_{j=1}^{n} \int_{-\tau}^{0} W_{j}(s+\theta) d v_{j}(\theta)\right) d s\right] .
\end{aligned}
$$

Choosing $\varepsilon=(4 \sqrt{2} D)^{-1}\left(2+\exp \left\{r \gamma_{r} \tau\right\}\right)^{-1}$ yields

$$
\begin{aligned}
& E \sup _{0 \leq \bar{\theta} \leq \tau}\left|\int_{k \tau}^{k \tau+\bar{\theta}} \frac{\partial W_{i}^{\mathrm{T}}}{\partial x} G(s) d B(s)\right| \\
& \leq\left(2 n+n \exp \left\{\gamma_{r} \tau\right\}\right)^{-1} \\
& \quad \times \sum_{j=1}^{n}\left(E\left\|W_{j, k \tau}\right\|+0.5 E\left\|W_{j,(k-1) \tau}\right\|\right) \\
& +16 D^{2} n\left(2+\exp \left\{\gamma_{r} \tau\right\}\right) \int_{k \tau}^{(k+1) \tau} \sum_{j=1}^{n}\left(E W_{j}(s)\right. \\
& \left.+\int_{-\tau}^{0} E W_{j}(s+\theta) d v_{j}(\theta)\right) d s .
\end{aligned}
$$


By condition (17) with $v(t)=0$, we compute that

$$
\begin{aligned}
& E \int_{k \tau}^{(k+1) \tau} W_{j}(s) d s \leq K \alpha_{j}^{r} \tau \exp \left\{-r \gamma_{r}\right\} k \tau, \\
& E \int_{k \tau}^{(k+1) \tau} \int_{-\tau}^{0} W_{j}(s+\theta) d v(\theta) \\
& \leq K \alpha_{j}^{r} \tau \exp \left\{r \gamma_{r} \tau\right\} \exp \left\{-r \gamma_{r}\right\} k \tau .
\end{aligned}
$$

Substituting (36) and (37) into (34) yields

$$
\begin{aligned}
& E\left\|W_{i, k \tau}\right\|=\left(2 n+n \exp \left\{r \gamma_{r} \tau\right\}\right)^{-1} \sum_{i=1}^{n} E\left\|W_{i, k \tau}\right\| \\
& +\left(4 n+2 n \exp \left\{r \gamma_{r} \tau\right\}\right)^{-1} \sum_{i=1}^{n} E\left\|W_{i,(k-1) \tau}\right\| \\
& +\sum_{i=1}^{n} C_{i} \exp \left\{-r \gamma_{r} t\right\},
\end{aligned}
$$

where $C_{i}=K \alpha_{i}^{r}+K \tau\left(r^{\prime-1} \sum_{j=1}^{n}\left(a_{i j}+b_{i j}\right) \alpha_{i}^{r}+\right.$ $r^{-1} \sum_{j=1}^{n} a_{i j} \alpha_{j}^{r}+r^{-1} \sum_{j=1}^{n} b_{i j} \alpha_{j}^{r} \exp r \gamma_{r} \tau+16 n D^{2}(2+$ $\left.\left.\exp r \gamma_{r} \tau\right) \sum_{j=1}^{n} \alpha_{j}^{r}\left(1+\exp r \gamma_{r} \tau\right)\right)$. Summing $E\left\|W_{i, k \tau}\right\|$ from 1 to $n$ yields

$$
\begin{aligned}
& \sum_{i=1}^{n} E\left\|W_{i, k \tau}\right\| \\
& =\left(2+\exp \left\{r \gamma_{r} \tau\right\}\right)^{-1} \sum_{i=1}^{n} E\left\|W_{i, k \tau}\right\|+\sum_{i=1}^{n} C_{i} \exp \left\{-r \gamma_{r} t\right\} \\
& \quad+\left(4+2 \exp \left\{r \gamma_{r} \tau\right\}\right)^{-1} \sum_{i=1}^{n} E\left\|W_{i,(k-1) \tau}\right\|,
\end{aligned}
$$

This implies for any $k=0,1, \cdots$,

$$
\begin{gathered}
\sum_{i=1}^{n} E\left\|W_{i, k \tau}\right\| \leq \frac{1}{2+2 \exp \left\{r \gamma_{r} \tau\right\}} \sum_{i=1}^{n} E\left\|W_{i,(k-1) \tau}\right\| \\
+\frac{1}{1-\left(2+\exp \left\{r \gamma_{r} \tau\right\}\right)^{-1}} \sum_{i=1}^{n} C_{i} \exp \left\{-r \gamma_{r} k \tau\right\}
\end{gathered}
$$

For any $\varepsilon \in\left(0, \gamma_{r}\right)$, it follows from Chebyshev inequality that

$$
\begin{aligned}
& P\left\{\left\|W_{i, k \tau}\right\|>C_{i} \exp \left\{-r\left(\gamma_{r}-\varepsilon\right) k \tau\right\}\right\} \\
& \quad \leq \exp \left\{r\left(\gamma_{r}-\varepsilon\right) k \tau\right\} E\left\|W_{i, k \tau}\right\| \\
& \quad \leq \exp \left\{r\left(\gamma_{r}-\varepsilon\right) k \tau\right\} \sum_{i=1}^{n} E\left\|W_{i, k \tau}\right\| .
\end{aligned}
$$

Setting $A_{k}=\left\{\left\|W_{i, k \tau}\right\|>\exp \left\{-p\left(\gamma_{r}-\varepsilon\right) k \tau\right\}\right\}$. Note that

$$
\begin{aligned}
& \exp \left\{r\left(\gamma_{r}-\varepsilon\right) k \tau\right\} \sum_{i=1}^{n} E\left\|W_{i, k \tau}\right\| \leq \frac{\exp \left\{r\left(\gamma_{r}-\varepsilon\right) \tau\right\}}{2+2 \exp \left\{r \gamma_{r} \tau\right\}} \\
& \times \exp \left\{r\left(\gamma_{r}-\varepsilon\right)(k-1) \tau\right\} \sum_{i=1}^{n} E\left\|W_{i,(k-1) \tau}\right\| \\
& +\frac{\sum_{i=1}^{n} C_{i}}{1-\left(2+\exp \left\{r \gamma_{r} \tau\right\}\right)^{-1}} \exp \{-r \varepsilon k \tau\} \\
& k=0,1, \cdots
\end{aligned}
$$

Let $v_{1}=\exp \left\{r\left(\gamma_{r}-\varepsilon\right) \tau\right\}\left(2+2 \exp \left\{r \gamma_{r} \tau\right\}\right)^{-1}<1, v_{2}=$ $C_{i}\left(1-\left(2+\exp \left\{r \gamma_{r} \tau\right\}\right)^{-1}\right)^{-1}$. Summing $\exp \left\{2\left(\gamma_{r}-\right.\right.$ $\varepsilon) k \tau\} \sum_{i=1}^{n} E\left\|V_{i, k \tau}\right\|$ from 0 to $\infty$ yields

$$
\begin{aligned}
\sum_{k=0}^{\infty} \exp \left\{r\left(\gamma_{r}-\varepsilon\right) k \tau\right\} \sum_{i=1}^{n} E\left\|W_{i, k \tau}\right\| \\
\leq v_{1} \sum_{k=0}^{\infty} \exp \left\{r\left(\gamma_{r}-\varepsilon\right) k \tau\right\} \sum_{i=1}^{n} E\left\|W_{i, k \tau}\right\| \\
\quad+v_{2} \frac{1}{1-\exp \{-r \varepsilon k \tau\}}+\sum_{i=1}^{n} E\left\|W_{i, 0}\right\|,
\end{aligned}
$$

which means $\sum_{k=0}^{\infty} \exp \left\{r\left(\gamma_{r}-\varepsilon\right) k \tau\right\} E\left\|W_{i, k \tau}\right\|<\infty$. The well-known Borel-Cantelli Lemma (see [16]) yields

$$
P\left(\bigcup_{k=1}^{\infty} \bigcap_{k=n}^{\infty} A_{k}^{c}\right)=1
$$

This implies for any $\omega \in \bigcup_{k=1}^{\infty} \bigcap_{k=n}^{\infty} A_{k}^{c}$, there exists an integer $N(\omega)$ such that for all $k \geq N(\omega)$, we have

$$
\sup _{k \tau \leq t \leq(k+1) \tau} W_{i}(t) \leq C_{i} \exp \left\{-r\left(\gamma_{r}-\varepsilon\right) k \tau\right\} \text { a.s. }
$$

For any $k \geq N_{0}, t \in[k \tau,(k+1) \tau]$, we have

$$
W_{i}(t) \leq C_{i} \exp \left\{\gamma_{r} \tau\right\} \exp \left\{-r \gamma_{r} t\right\} \text { a.s. }
$$

Hence we have assertion (32) as desired. The proof is completed.

Remark 5: Noting that the condition (30) is specialized for the almost surely exponentially stable. For those systems satisfying LGC along with the power function type bilateral condition posed on Lyapunov function, the $\mathscr{L}$-operator differential inequality (15) can be re- 
placed by the weaker inequality as following

$$
\begin{aligned}
& E \mathscr{L} W_{i}\left(t, x_{t}\right) \\
& \leq-\mu_{i} E W_{i}(t, x(t))+\sum_{j=1}^{n} b_{i j}\left(E W_{i}(t, x(t))\right)^{1 / r^{\prime}} \\
& \quad \times \sup _{-\tau \leq \theta \leq 0}\left(E W_{j}(t+\theta, x(t+\theta))\right)^{1 / r} \\
& \quad+\sum_{j=1}^{n} a_{i j}\left(E W_{i}(t, x(t))\right)^{1 / r^{\prime}}\left(E W_{j}(t, x(t))\right)^{1 / r} .
\end{aligned}
$$

When $a_{i j}=0$ and $p=1$ or $p=2$, the inequalities (39) have the same structure as the inequalities presented by Shen and Wang in [22] in the constant coefficient case.

Remark 6: Noting, for $G=0$, the SFDS (29) degenerate into the deterministic functional differential system. In this case, the boundedness of $p$ th moment and the $\mathscr{F}_{t}$-adapted of solution $x(t)$ are not required. Taking $r=1$, the $\mathscr{L}$-operator differential inequality (39) has a concise form for the deterministic system as following:

$$
\begin{gathered}
D^{+} W_{i}(t, x(t)) \leq-\mu_{i} W_{i}(t, x(t))+\sum_{j=1}^{n} a_{i j} W_{j}(t, x(t)) \\
+\sum_{j=1}^{n} b_{i j} \sup _{-\tau \leq \theta \leq 0} W_{j}(t+\theta, x(t+\theta)) .
\end{gathered}
$$

Setting $p_{i i}=a_{i i}-\mu_{i}, p_{i j}=a_{i j}, i \neq j, q_{i j}=b_{i j}$, the inequality (40) becomes the vector $\mathscr{L}$-operator differential inequality presented in [27] without external input.

We now use Theorem 3.3 to establish a useful corollary.

Corollary 3.3: Let all the assumptions of Theorem 3.2 hold. Moreover, if there exists $\left(c_{1}, \cdots, c_{n}\right) \gg$ $0,\left(p_{1}, \cdots, p_{n}\right) \gg 0$ such that

$$
c_{i}\left|u_{i}\right|^{p_{i}} \leq W_{i}(t, u), t \geq 0, i=1, \cdots, n .
$$

Then the global solution $x(t ; \xi)$ to system (27) has the following property

$$
\begin{aligned}
& \limsup _{t \rightarrow \infty} \frac{\log E\left|u_{i}(t)\right|^{p_{i}}}{t} \leq-r \gamma_{r}, \\
& \limsup _{t \rightarrow \infty} \frac{\log \left|u_{i}(t)\right|}{t} \leq-\frac{r \gamma_{r}}{p_{i}} \text { a.s., } i=1, \cdots, n .
\end{aligned}
$$

Remark 7: Compared with existing criteria in [10], [11], [17], [21], our criteria show that different component might has different degree of stability in moment sense and almost sure sense. Hence, our criteria provided a more detailed description on the asymptotic behavior of system than that of these existing criteria.

Remark 8: It is well known that the almost sure exponential stability can be acquired by the moment exponential stability under the LGC imposing on both drift coefficient and diffusion coefficient in [3], [12] and [16]. It was $\mathrm{Wu}$ and $\mathrm{Hu}$ who first pointed out that the LGC imposing on both drift coefficient is not necessary [26]. In comparison with $\mathrm{Wu}$ and $\mathrm{Hu}$ [26], our criteria further point out that that the LGC imposing on diffusion coefficient can be replaced by a more generalized condition (30).

\section{Application and Numerical Examples}

\subsection{Neural Networks}

Recurrent neural networks (RNNs) have been widely applied in many fields (see [34], [35], [36] ) owing to the pioneering work of Hopfield [33]. It is well known that neural networks are often affected by external inputs, such as perturbations in control or errors on observation. Recently, we have also noticed that more and more scholars begin to discuss the $p$-th moment ISS of neural networks by means of the vector Lyapunov function methods, see, e.g., [4], [31]. Moreover, Haykin [32] points out that in real nervous systems, synaptic transmission “. . . is a noisy process brought on by random fluctuations from the release of neurotransmitters, and other probabilistic causes". Thus, it is interesting to study how noise affect the ISS of neural networks. The approach used in the current paper is to view neural networks as nonlinear dynamical systems with intrinsic noise and external input, described by the following stochastic differential delay system

$$
\begin{gathered}
d x(t)=(-D x(t)+A f(x(t))+B f(x(t-\tau(t)))+u(t)) d t \\
+\sigma(x(t)) d B(t)
\end{gathered}
$$

where $x(t)=\left(x_{1}(t), x_{2}(t), \cdots, x_{n}(t)\right)^{T} \in R^{n}$ are the state vector, $f(x(t))=\left(f_{1}\left(x_{1}(t)\right), \cdots, f_{n}\left(x_{n}(t)\right)\right)^{T}, f(x(t-$ $\tau(t)))=\left(f_{1}\left(x_{1}\left(t-\tau_{1}(t)\right)\right), f_{2}\left(x_{2}\left(t-\tau_{2}(t)\right)\right), \cdots, f_{n}\left(x_{n}(t-\right.\right.$ $\left.\left.\left.\tau_{n}(t)\right)\right)\right)^{T}$ are the neuron activation function with $f_{i}(0)=$ $0, \tau_{i}(t)>0$ are the transmission delay. Moreover, $D=$ $\operatorname{diag}\left(d_{1}, \cdots, d_{n}\right)$ is a self-feedback connection weight matrix with $d_{i}>0, A$ and $B$ are connection weight matrices associated without delay and with delay, respectively. $\sigma(x)=\operatorname{diag}\left(\sigma_{1} x_{1}, \cdots, \sigma_{n} x_{n}\right)$ is the noise intensity matrix. System (41) can be regarded as the stochastically perturbed system of corresponding deterministic RNN. Now we present need the following assumptions which we will use later. 
Assumption 2: For $i=1, \cdots, n$, there exists $\kappa_{i}>0$ such that $\forall \theta_{1}, \theta_{2} \in R$

$$
0 \leq \frac{f_{i}\left(\theta_{1}\right)-f_{i}\left(\theta_{2}\right)}{\theta_{1}-\theta_{2}} \leq \kappa_{i},
$$

for all $i=1,2, \cdots, n$. Denoted $K=\operatorname{diag}\left(\kappa_{1}, \cdots, \kappa_{n}\right)$.

Noting that the system (41) is a weak nonlinear system and the structure of every component state is alike, choosing $\left|x_{i}\right|^{p}$ for every state $x_{i}$. Thus the vector Lyapunov function for system (41) is $\left(\left|x_{1}\right|^{p},\left|x_{2}\right|^{p}, \cdots,\left|x_{n}\right|^{p}\right)$. Then the following Theorem 4.1 for system (41) follows from Theorem 3.1 straightforward.

Theorem 4.1: Let Assumption 2 hold. The system (41) is said to be $p$-th moment exponentially input-to-state stable provided $\Lambda(p, \sigma)-(|\bar{A}|+|B|) K$ is a nonsingular M-matrix with $\Lambda(p, \sigma)=D-0.5 p \operatorname{diag}\left(\sigma_{1}^{2}, \cdots, \sigma_{n}^{2}\right)$.

Example 4.1: Consider a delayed two-neuron network as follows:

$$
\left\{\begin{array}{c}
d x_{1}(t)=\left(-1.2 x_{1}(t)-0.7 f_{1}\left(x_{1}(t)\right)\right. \\
+0.8 f_{2}\left(x_{2}(t)\right)+0.1 f_{1}\left(x_{1}(t-\tau(t))\right) \\
\left.+0.3 f_{2}\left(x_{2}(t-\tau(t))\right)+2|\sin 8 t|\right) d t \\
+\sqrt{0.1} x_{1}(t) d B_{1}(t), \\
d x_{2}(t)=\left(-2 x_{2}(t)+0.4 f_{1}\left(x_{1}(t)\right)\right. \\
-0.5 f_{2}\left(x_{2}(t)\right)+0.3 f_{1}\left(x_{1}(t-\tau(t))\right) \\
\left.-0.5 f_{2}\left(x_{2}(t-\tau(t))\right)+|\cos 4 t|\right) d t \\
+\sqrt{0.5} x_{2}(t) d B_{2}(t) .
\end{array}\right.
$$

where $f(x)=(\exp \{2 x\}+\exp \{-2 x\})^{-1}(\exp \{2 x\}-$ $\exp \{-2 x\})$, the time-varying delay $\tau(t)=1.8|\sin (t)|$. Applying Itô formula to $W_{i}(x)=\left|x_{i}\right|^{p}$ yields

$$
\begin{aligned}
\mathscr{L} & V_{1}\left(x_{t}, u(t)\right) \leq-1.2 p\left|x_{1}(t)\right|^{p}+0.8 p\left|x_{1}(t)\right|^{p-1}\left|x_{2}(t)\right| \\
& +0.1 p\left|x_{1}(t)\right|^{p-1}\left|x_{1}(t-\tau(t))\right|+0.3 p\left|x_{1}(t)\right|^{p-1} \\
& \times\left|x_{2}(t-\tau(t))\right|+2 p\left|x_{1}(t)\right|^{p-1}|\sin 8 t|, \\
\mathscr{L} & V_{2}\left(x_{t}, u(t)\right) \leq-2 p\left|x_{2}(t)\right|^{p}+0.4 p\left|x_{2}(t)\right|^{p-1}\left|x_{1}(t)\right| \\
& +0.3 p\left|x_{2}(t)\right|^{p-1}\left|x_{1}(t-\tau(t))\right|+0.5 p\left|x_{2}(t)\right|^{p-1} \\
& \times\left|x_{2}(t-\tau(t))\right|+p\left|x_{2}(t)\right|^{p-1}|\cos 4 t| .
\end{aligned}
$$

The parameter matrix in Theorem 4.1 become as:

$$
\begin{aligned}
\Lambda(p, \sigma) & =\left(\begin{array}{cc}
1.2-0.05(p-1) & 0 \\
0 & 2-0.25(p-1)
\end{array}\right) . \\
|\bar{A}| & =\left(\begin{array}{cc}
0 & 0.8 \\
0.4 & 0
\end{array}\right), \quad|B|=\left(\begin{array}{ll}
0.1 & 0.3 \\
0.3 & 0.5
\end{array}\right)
\end{aligned}
$$

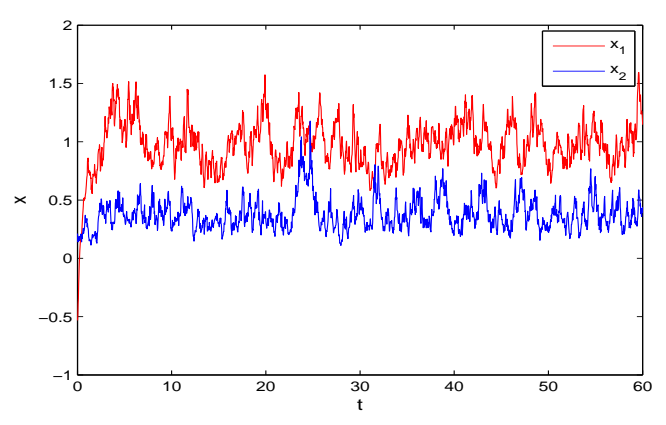

The transient state of $\left(x_{1}(t), x_{2}(t)\right)$ with $\tau(t)=1.8|\sin (t)|$ in Example 4.1.

By Theorem 2.10 in [15], we can show that $\Lambda_{p}-\left(A_{p}+B_{p}\right)$ is a nonsingular M-matrix for any $p \in(0,4.3674)$. According to Theorem 3.2 and Corollary 3.2 , the system (42) is $p$ th moment exponentially input-to-state stable.

Remark 9: It is obvious that the system (42) is mean square exponential ISS. Now we process to apply the existing results in [31] to show the effectiveness of our criteria. For system (42), the parameters matrix $T$ and $S$ become

$$
T=\left(\begin{array}{cc}
-0.1 & 0.8 \\
0.4 & -2.3
\end{array}\right), \quad S=\left(\begin{array}{ll}
0.1 & 0.3 \\
0.3 & 0.5
\end{array}\right)
$$

Simple computations show that $-(T+S)$ is not a nonsingular M-matrix, which means the Corollary 3.1 in [31] could not be applied to the system.

\subsection{A numerical example}

Example 4.2: Consider a two-dimensional nonautonomous system with the form

$$
\left\{\begin{array}{l}
d x_{1}(t)=\sqrt{\left|x_{1}(t)\right|}\left[\left(-2 \operatorname{sign} x_{1}(t) \sqrt{\left|x_{1}(t)\right|}\right.\right. \\
\left.+0.5|\sin t| x_{2}(t)+0.5 \int_{-1}^{0} x_{2}(t+\theta) d \eta(\theta)\right) d t \\
\left.+\sqrt{0.5} \operatorname{sign} x_{1}(t) \sqrt{\left|x_{1}(t)\right|} d B_{1}(t)\right] \\
d x_{2}(t)=\left(-2.4 x_{2}(t)+0.6\left(1+e^{-t}\right) \sqrt{\left|x_{1}(t)\right|}\right. \\
\left.+\sqrt{\int_{-1}^{0}\left|x_{1}(t+\theta)\right| d \eta(\theta)}\right) d t+\sqrt{0.5} x_{2}(t) d B_{2}(t) .
\end{array}\right.
$$

where $\eta(\theta)=(1-\exp \{-1\})^{-1} \exp \{\theta\}$ is defined on $[-1,0]$.

Such system and its alike can be seen as generalized stochastic power law logistic model (see [? ] and the references therein), and could been used to study the 
extinction of population systems under noise. The numerical system (43) is a strong nonlinear system, of which every component equation have different coefficient structure. Thus different Lyapunov functions should be chosen for every state component. Based on detailed observation, $V_{1}=\left|x_{1}\right|^{3}$ is chosen for $x_{1}$ and $V_{2}=\left|x_{2}\right|^{6}$ is chosen for $x_{2}$. Thus

$$
\left\{\begin{aligned}
& \mathscr{L} V_{1}\left(t, x_{t}\right) \\
\leq & -5.4\left|x_{1}(t)\right|^{3}+1.5\left(\left|x_{1}(t)\right|^{3}\right)^{5 / 6}\left(\left|x_{2}(t)\right|^{6}\right)^{1 / 6} \\
& +1.5\left(\left|x_{1}(t)\right|\right)^{5 / 6}\left(\int_{-1}^{0}\left|x_{2}(t+\theta)\right|^{6} d \eta(\theta)\right)^{1 / 6} \\
& \mathscr{L} V_{2}\left(t, x_{t}\right) \\
\leq & -11.4 x_{6}^{2}(t)+7.2\left(\left|x_{2}(t)\right|^{6}\right)^{5 / 6}\left(\left|x_{1}(t)\right|^{3}\right)^{1 / 6} \\
& +6\left(\left|x_{2}(t)\right|^{6}\right)^{5 / 6}\left(\int_{-1}^{0}\left|x_{1}(t+\theta)\right|^{3} d \eta(\theta)\right)^{1 / 6}
\end{aligned}\right.
$$

Under such vector Lyapunov function, the system (43) can be transformed into a system with negative $\mathrm{M}$ matrix system, and the parameter in Theorem 3.2 can be rewritten as

$$
\mu=\left(\begin{array}{cc}
5.4 & 0 \\
0 & 11.4
\end{array}\right), \quad A=\left(\begin{array}{cc}
0 & 1.5 \\
7.2 & 0
\end{array}\right), \quad B=\left(\begin{array}{cc}
0 & 1.5 \\
6 & 0
\end{array}\right) .
$$

By simple computing, we derive that $\mu-(A+B)$ is a nonsingular M-matrix, and $\gamma_{6}=0.2468$ with choosing $\alpha_{1}=1, \alpha_{2}=1.5$.

Remark 10: It is worth noting that the vector operator differential inequality (15) is more compatible with the $p$ th moment exponential ISS for stochastically perturbed neural network and stochastically perturbed generalized ecological systems than those in [16], [27], [29].

Based on the vector operator differential inequality, the choosing of Lyapunov function becomes easier than those criteria describe by operator differential inequality. When choosing the classical quadratic function $W_{1}=x_{1}^{2}+x_{2}^{2}$, the $\mathscr{L} W_{1}$ has the form

$$
\begin{aligned}
\mathscr{L} & W_{1}\left(t, x_{t}\right) \\
\leq & -3.5 u_{1}^{2}(t)-4.3 u_{2}^{2}(t)+\left|x_{1}(t)\right|^{3 / 2}\left|x_{1}(t)\right| \\
& +0.5\left|x_{1}(t)\right|^{3 / 2} \int_{-1}^{0}\left|x_{2}(t+\theta)\right| d \eta(\theta) \\
& +2.4 \sqrt{\left|x_{1}(t)\right| \mid} x_{2}(t)\left|+2 \int_{-1}^{0}\right| x_{1}(t+\theta) \mid d \eta(\theta) .
\end{aligned}
$$

Noting that the higher order of polynomial, such as $\left|x_{1}(t)\right|^{3 / 2}\left|x_{1}(t)\right|, 0.5\left|x_{1}(t)\right|^{3 / 2} \int_{-1}^{0}\left|x_{2}(t+\theta)\right| d \eta(\theta)$, appear on the right-hand side of inequality (45) and this prevents the exiting results, such as, Theorem 8.7 in [16] from being used for $W_{1}=x_{1}^{2}+x_{2}^{2}$.
On the other hand, applying the Itô formula to $W_{2}=$ $\left|x_{1}\right|^{3}+x_{2}^{6}$ yields

$$
\begin{aligned}
& \mathscr{L} W_{2}\left(t, x_{t}\right) \\
& \leq 3 \operatorname{sign} x_{1}\left|x_{1}\right|^{2}\left(-2 x_{1}(t)+0.5 \sqrt{\left|x_{1}\right|}\left|x_{2}(t)\right|\right. \\
& \left.\quad+0.5 \sqrt{\left|x_{1}(t)\right|} \int_{-1}^{0}\left|x_{2}(t+\theta)\right| d \eta(\theta)\right)-14.4 x_{6}^{2}(t) \\
& \quad+\left.7.2 \sqrt{\left|x_{1}(t)\right| \mid} x_{2}(t)\right|^{5}+6\left(\int_{-1}^{0}\left|x_{1}(t+\theta)\right|^{3} d \eta(\theta)\right)^{1 / 6} \\
& \quad \times\left|x_{2}(t)\right|^{5}+0.6\left|x_{1}(t)\right|^{3}+3 x_{2}^{6}(t) .
\end{aligned}
$$

By the Young inequality (stated in Theorem 3.3), we can show we have

$$
\begin{aligned}
\mathscr{L} W_{2}\left(t, x_{t}\right) & \leq-0.15 x_{2}^{6}(t)+0.25 \int_{-1}^{0}\left|x_{2}(t+\theta)\right|^{6} d \eta(\theta) \\
& -1.7\left|x_{1}(t)\right|^{3}+\int_{-1}^{0}\left|x_{1}(t+\theta)\right|^{3} d \eta(\theta) .
\end{aligned}
$$

Since $0.25>0.15$, the existing stability criteria in [10], [11], [17], [21], based on the SLF are invalid for $W_{2}=$ $\left|x_{1}\right|^{3}+x_{2}^{6}$.

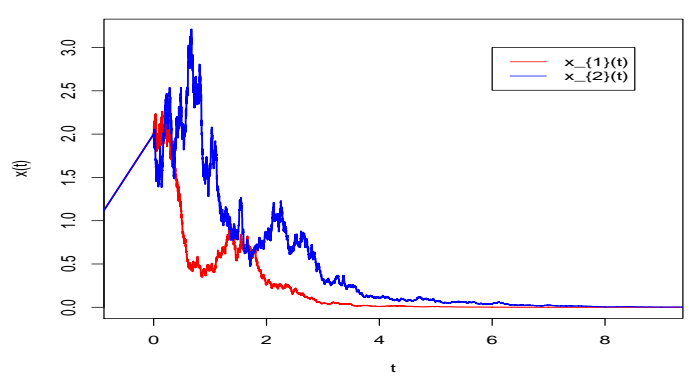

The transient state of $\left(x_{1}(t), x 2(t)\right)$ in Example 4.2.

\section{Conclusion}

This paper have studied the $p$ th moment exponential ISS for SFDSs. Firstly, by utilizing stochastic analysis techniques and VLF, a vector Razumikhin-type theorem has been derived, which might have a compatible structure with neural networks systems. Secondly, with the help of the established Razumikhin-type theorem, sufficient criteria on $p$ th moment exponential ISS have been established. Finally, for the systems with zero input, the established criteria improved the conditions imposed on exponential stability. Two numerical examples are provided to illustrate the superiority and effectiveness of the proposed results. 


\section{Acknowledgement}

The project reported here is supported by the National Science Foundation of China (Grant Nos. 613040706), the China Postdoctoral Science Foundation(Grant Nos. 2016M601698) and the Fundamental Research Fund$\mathrm{s}$ for the Central Universities of China. (Grant No.2015B19814).

\section{Reference}

[1] L. Huang, X. Mao, and F. Deng, Stability of hybrid stochastic retarded systems, IEEE Trans. Circuits Syst. I, 55(11), pp. 34133420,2008

[2] L. Huang and X. Mao, On input-to-state stability of stochastic retarded systems with Markovian switching, IEEE Trans. Automat. Contr., 54(8), pp. 1898-1902, 2009.

[3] S. Jankovic, J. Randjelovic, and M. Jovanovic, Razumikhin-type exponential stability criteria of neutral stochastic functional differential equations, J. Math. Anal. Appl., 355(2), pp. 811-820, 2009.

[4] Z. Li, L. Liu, and Q. Zhu, Mean-square exponential input-tostate stability of delayed Cohen-Grossberg neural networks with Markovian switching based on vector Lyapunov functions, Neural Networks, 84, pp. 39-46, 2016.

[5] S. Liu, J. Zhang, and Z. Jiang, A notion of stochastic input-tostate stability and its application to stability of cascaded stochastic nonlinear systems, Acta. Math. Appl. Sin-E, 24(1), pp. 141156, 2008.

[6] B. Liu and D. Hill, Input-to-state stability for discrete time-delay systems via the Razumikhin technique, Syst. \& Control Lett., 58(8), pp. 567-575, 2009.

[7] L. Liu, Y. Shen, and F. Jiang, The almost sure asymptotic stability and pth moment asymptotic stability of nonlinear stochastic differential systems with polynomial growth, Asian Journal of Control, 14(3), pp. 859-867, 2012.

[8] L. Liu and Y. Shen, Noise suppresses explosive solutions of differential systems with coefficients satisfying the polynomial growth condition, Automatica, 48, pp. 619-624, 2012.

[9] L. Liu, New criteria on Exponential stability for stochastic delay differential systems based on vector Lyapunov function, IEEE Trans. on Systems, Man, and Cybernetics: Systems, 2016.

[10] Q. Luo, X. Mao, and Y. Shen, New criteria on exponential stability of neutral stochastic differential delay equations, Syst. \& Control Lett., 55(10), pp. 826-834, 2006.

[11] Q. Luo, X. Mao, and Y. Shen, Generalised theory on asymptotic stability and boundedness of stochastic functional differential equations, Automatica, 47(9), pp. 2075-2081, 2011.

[12] X. Mao, Razumikhin-type theorems on exponential stability of stochastic functional differential equations, Stoch. Proc. Appl., 65(2), pp. 233-250, 1996

[13] X. Mao, Razumikhin-type theorems on exponential stability of neutral stochastic functional differential, SIAMJ. on Math. Anal., 28(2), pp. 389-401, 1997.

[14] X. Mao, A. Rodkina, and N. Koroleva, Razumikhin-type theorems for neutral stochastic functional differential equations, Functional Differential Equations, 5, pp. 195-211, 1998.

[15] X. Mao and C. Yuan, Stochastic Differential Equations with Markovian Switching, London, U.K.: Imperial College Press, 2006.

[16] X. Mao, Stochastic Differential Equations and Applications, Horwood: Chichester, 2007.
[17] X. Mao, Y. Shen, and C. Yuan, Almost surely asymptotic stability of neutral stochastic differential delay equations with Markovian switching, Stoch. Proc. Appl., 118(8), pp. 1385-1406, 2008.

[18] C. Ning, Y. He, and M. Wu, Improved Razumikhin-type theorem for input-to-state stability of nonlinear time-delay systems, IEEE Trans. Automat. Contr., 59(7), pp. 1983-1988, 2014.

[19] P. Pepe and Z. Jiang, A Lyapunov-Krasovskii methodology for ISS and iISS of time-delay systems", Syst. \& Control Lett., 55(12), pp. 1006-1014, 2006.

[20] S. Nersesov and W. Haddad, On the stability and control of nonlinear dynamical systems via vector Lyapunov functions, IEEE Trans. Automat. Contr., 51(20), pp. 203-215, 2006.

[21] Y. Shen, Q. Luo, and X. Mao, The improved LaSalle-type theorems for stochastic functional differential equations, J. Math. Anal. Appl., 318(1), pp. 134-154, 2006.

[22] Y. Shen and J. Wang, Almost sure exponential stability of recurrent neural networks with Markovian switching, IEEE Trans. Neural Netw., 20(5), pp. 840-855, 2009.

[23] E. Sontag, Smooth stabilization implies coprime factorization, IEEE T. Automat. Contr., 34(4), pp. 435-443, 1989.

[24] J. Tsinias, Stochastic input-to-state stability and applications to global feedback Stabilization, Int. J. Control, 71(5), pp. 907930, 1998.

[25] X. Wang, Q. Guo, and D. Xu, Exponential p-stability of impulsive stochastic Cohen-Grossberg neural networks with mixed delays, Math. Comput. Simulat., 79(5), pp. 1698-1710, 2009.

[26] F. Wu and S. Hu, Razumikhin-type theorems on general decay stability and robustness for stochastic functional differential equations, Int. J. Robust. Nonlin., 22, pp. 763-777, 2012.

[27] D. Xu and Z. Yang, Impulsive delay differential inequality and stability of neural networks, J. Math. Anal. Appl., 305(1), pp. 107-120, 2005

[28] D. Xu, W. Zhu, and S. Long, Global exponential stability of impulsive integro-differential equation, Nonlinear Anal. TMA., 64(12), pp. 2805-2816, 2006.

[29] L. Xu and D. Xu, P-attracting and p-invariant sets for a class of impulsive stochastic functional differential equations, Comput. Math. Appl., 57(1), pp. 54-61, 2009.

[30] P. Zhao, W. Feng, and Y. Kang, Stochastic input-to-state stability of switched stochastic nonlinear systems, Automatica, 48(10), pp. 2569-2576, 2012.

[31] W. Zhou, L. Teng, and D. Xu, Mean-square exponentially inputto-state stability of stochastic Cohen-Grossberg neural networks with time-varying delays, Neurocomputing, 153, pp. 54-61, 2015.

[32] S. Haykin, Neural Networks, Prentice-Hall, NJ, 1994.

[33] J. Hopfield, "Neural networks and physical systems with emergent collective computational abilities", Proc. Nat. Acad. Sci. (Biophysics), vol. 79, pp. 2554-2558, Apr. 1982.

[34] Long Cheng,Zeng-Guang Hou, Yingzi Lin, Min Tan, Wenjun Chris Zhang, and Fang-Xiang Wu, Recurrent Neural Network for Non-Smooth Convex Optimization Problems With Application to the Identification of Genetic Regulatory Networks IEEE Transactions on Neural Networks Volume: 22, Issue: 5, May 2011 Page(s): 714 - 726

[35] Q. Liu and J. Wang, A one-layer projection neural network for nonsmooth optimization subject to linear equalities and bound constraints, IEEE Transactions on Neural Networks and Learning Systems, vol. 24, no. 5, pp. 812C824, May 2013.

[36] Zhigang Zeng, Jun Wang, Design and analysis of high-capacity associative memories based on a class of discrete-time recurrent neural networks, IEEE Transactions on Systems, Man, and Cybernetics, Part B: Cybernetics, Vol.38, No.6, pp.1525-1536, 2008. 
[37] V.B. Kolmanovskii and V.R. Nosov, Stability of Functional Differential Equations (Academic Press, New York, 1986).

[38] On the stability of systems with a delay BS Razumikhin - Prikl. Mat. Mekh, 1956

[39] Application of Liapunov's method to problems in the stability of systems with a delay BS Razumikhin - Automat. i Telemeh, 1960

[40] Stability of motion: applications of Lyapunov's second method to differential systems and equations with delay NN Krasovskii, J McCord, J Gudeman - 1963 - Stanford university press

[41] Bahar, A., Mao, X. (2008). Persistence of stochastic power law logistic model. J. Appl. Probab. Stat., 3(1), 37C43. 